\title{
Tissue distributions of antiviral drugs impact on their capabilities of reducing viral loads in COVID-19 treatment
}

\author{
Yan WANG ${ }^{1}$ and Lei Chen ${ }^{2}$ \\ ${ }^{1}$ Shenzhen Institutes of Advanced Technology Chinese Academy of Sciences \\ ${ }^{2}$ Rutgers University
}

July 7,2020

\begin{abstract}
Background and Purpose Previously we reported our hypothesis that the high distribution of antiviral drugs in the lung is a key factor that results in reducing viral loads in COVID-19 patients. So far, chloroquine, lopinavir, hydroxychloroquine, azithromycin, favipiravir, ribavirin, darunavir, remdesivir, and umifenovir have been tested in COVID-19 clinical trials. Here we validate our hypothesis by comparing the pharmacokinetics profiles of these drugs and their capabilities of reducing viral load in clinical trials. Experimental approach The RNA-seq data were obtained from public database and re-analyzed and visualized by Single Cell Portal and Seurat. The tissue/plasma ratio of antiviral drugs were calculated by AUC or Mean values that were compiled from publications. Key Results High expression of both ACE2 and TMPRSS2 makes the lung and intestine vulnerable to SARS-CoV-2. Hydroxychloroquine, chloroquine, and favipiravir, which were highly distributed to the lung, were reported to reduce viral loads in respiratory tract of COVID-19 patients. Conversely, drugs with poor lung distributions, including lopinavir/ritonavir, umifenovir and remdesivir, were insufficient to inhibit SARS-CoV-2 replication. Lopinavir/ritonavir might inhibit SARS-CoV-2 in the GI tract according to their distribution profiles. Conclusion and Implications The antiviral drugs should be distributed straight to or accumulate in the lung for reducing viral loads in respiratory tract of COVID-1 9 patients. Additionally, to better evaluate antiviral drugs that target the intestine, the stool samples should also be collected for viral RNA test in the future.
\end{abstract}

\section{Hosted file}

BJP+manuscript.docx available at https://authorea.com/users/311576/articles/467726-tissuedistributions-of-antiviral-drugs-impact-on-their-capabilities-of-reducing-viral-loadsin-covid-19-treatment 